\title{
BLOOD LEVELS OF 17-HYDROXYCORTICOSTEROIDS FOLLOWING THE ADMINISTRATION OF ADRENAL STEROIDS AND THEIR RELATION TO LEVELS OF CIRCULATING LEUKOCYTES ${ }^{1}$
}

\author{
By DON H. NELSON, AVERY A. SANDBERG, ${ }^{2}$ J. G. PALMER, ${ }^{8}$ AND \\ FRANK H. TYLER
}

\begin{abstract}
(From the Departments of Biochemistry and Medicine of the University of Utah College of Medicine, Salt Lake City, Utah)
\end{abstract}

(Submitted for publication May 27, 1952; accepted July 16, 1952)

\section{INTRODUCTION}

In 1944 Dougherty and White (1) reported the occurrence of neutrophilia and lymphopenia following the administration of $\mathrm{ACTH}$ or adrenal cortical extracts to mice. In 1948 these findings were confirmed in man by Hills, Forsham and Finch (2), who noted, in addition, a pronounced fall in eosinophils. These changes in the blood have been used widely as an index of adrenal cortical activity. With the development of a method for the determination of 17-hydroxycorticosteroids in small quantities of blood or plasma (3) it has become possible to determine the relationship of the changes in blood steroid levels to the changes in leukocytes.

In order to investigate this relationship, standard doses of various corticosteroids were administered to normal individuals and the alterations in blood 17-hydroxycorticosteroids and in leukocytes were observed at intervals thereafter.

\section{METHODS}

Compound E (17-hydroxy-11-dehydrocorticosterone), compound F (17-hydroxycorticosterone), and compound S (17-hydroxy-11-desoxycorticosterone) were administered as the free alcohols and as the acetate esters. All subjects used were normal young adult males who were fasting at the time the steroids were given, and for at least three hours thereafter. When administered orally, the compounds were mixed with fruit juice, with the exception of compound $\mathrm{E}$ acetate, which was given as tab-

1 This investigation was supported in part by a research grant from the National Institute of Arthritis and Metabolic Diseases of National Institutes of Health, Public Health Service, and in part by a contract (DA-49-007MD-136) between the University of Utah and the Department of the Army.

2 Postdoctorate Research Fellow of the National Institutes of Health.

${ }^{3}$ Fellow, American Cancer Society. lets to two subjects (C. F. and T. O.). When administered intramuscularly, the compounds were in the form of a commercially available saline suspension except as indicated in the tables. Generally, the steroids were administered between 8:00 and 9:00 a.m. Blood was drawn for the steroid and blood cell determinations immediately prior to administration, and at one, four, eight, and 24 hours thereafter. These intervals were selected after preliminary studies in which samples were drawn at one-half hour intervals had demonstrated that the serum steroid peak occurred at approximately one hour and the previous observation of maximum cellular effects at approximately four hours was confirmed. Approximately $30 \mathrm{ml}$. of blood were drawn at each sampling. Heparin was used as an anticoagulant for the steroid determination and mixed oxalate for the cellular studies.

Determination of 17-hydroxycorticosteroids was carried out by the method of Nelson and Samuels (3). Ten milliliter samples of plasma were generally used for the determination. The Florisil chromatographic column was employed for purification and the micromodification of the color reaction of Porter and Silber was used for quantitation (4). Compounds $E, F$, and $S$ give this reaction. All levels of 17-hydroxycorticosteroids given are expressed in terms of $\mu \mathrm{gm}$. per $100 \mathrm{ml}$. of plasma.

Eosinophil counts were performed in duplicate by the method of Randolph (5). Total leukocyte counts were done in duplicate by routine methods and the average of the two counts was used. Absolute neutrophil and lymphocyte counts were calculated from the total leukocyte and differential counts. The latter were made from cover-slip blood films stained with Wright's stain, on which 500 cells were enumerated.

\section{RESULTS}

Figure 1 shows a representative response of leukocytes and plasma 17-hydroxycorticosteroid levels following the administration of $200 \mathrm{mgm}$. of compound $\mathrm{F}$ acetate orally. The data on all seven subjects who received this dose of compound $F$ acetate are shown in Table I. There was in each instance a marked rise in plasma levels of corticosteroids which reached a peak approxi- 


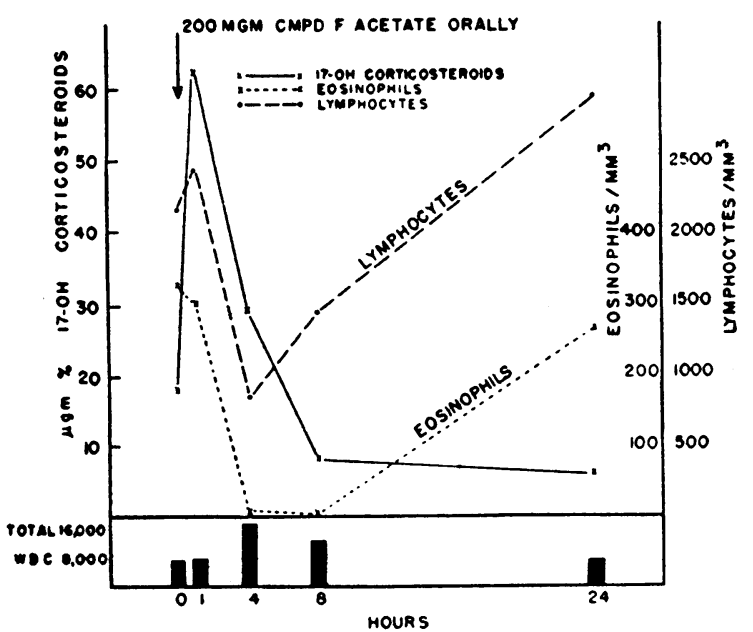

Fig. 1. The Effects of 200 mgm. of F Acetate Grven Orally on Blood 17-Hydroxysteroid LeVEls aNd Circulating White Blood Cell levels

Note that although the peak of corticosteroid level occurred at one hour, only negligible changes had appeared in the cellular elements at that time. At four hours when the steroid level had fallen sharply, the maximum effect on cellular elements made its appearance.

mately one hour after the steroid was given. The level then fell rapidly and returned to approximately normal at the end of eight hours. Although the highest concentration of steroids was reached at about one hour, the maximum change in leukocytes was not seen until four or eight hours after ingestion of the compound. In every instance a decrease in lymphocytes and eosinophils occurred as well as a pronounced rise in neutrophils; the result was a rise of the total white count.

A single subject was given oral doses of compound $F$ acetate ranging from 12.5 to $200 \mathrm{mgm}$. in order to determine the relation of dose to the response obtained. The experiments were carried out at approximately five-day intervals to minimize the effect of repeated dosage in the same subject. The results obtained are illustrated in Figure 2. When the levels at four hours are considered, it appears that the magnitude of the response in 17-hydroxycorticosteroids is progressively greater and more prolonged as the dosage of compound $\mathrm{F}$ is increased. Eosinophil levels fell at all dosage levels. With each increase in compound $\mathrm{F}$ a greater fall in eosinophils occurred until doses of 100 to $200 \mathrm{mgm}$. were given. With the latter doses, the eosinophils remained at very low levels for longer periods of time. Lymphocytes decreased with doses of $25 \mathrm{mgm}$. or more, the amount and duration of the decrease being roughly correlated with the dosage. Increases in neutrophils were also seen at each dose level but this was not correlated with the amount of compound given.

The effects of single injections of $200 \mathrm{mgm}$. of compound $\mathrm{F}$ acetate given intramuscularly to four individuals are shown in Table II. There was no rise in blood levels of corticosteroids and no significant effect upon the leukocytes.

TABLE I

Administration of 17-hydroxycorticosterone (Compound $F$ ) acetate, $200 \mathrm{mgm}$. orally

\begin{tabular}{|c|c|c|c|c|c|c|}
\hline \multirow{2}{*}{$\begin{array}{l}\text { Sub- } \\
\text { ject }\end{array}$} & \multirow{2}{*}{$\begin{array}{c}\text { Time } \\
\text { in } \\
\text { hours }\end{array}$} & \multirow{2}{*}{$\begin{array}{c}17-\mathrm{OH} \\
\text { steroid } \\
\text { levels, } \\
\gamma / 100 \mathrm{ml} . \\
\text { of plasma }\end{array}$} & \multirow{2}{*}{ WBC } & PMN & Lymph & \multirow{2}{*}{ EOS } \\
\hline & & & & \multicolumn{2}{|c|}{$\begin{array}{l}\text { Absolute number } \\
\text { per mm.? }\end{array}$} & \\
\hline M. C. & $\begin{array}{r}0 \\
\frac{1}{2} \\
1 \\
2 \\
4 \\
6 \\
8 \\
24\end{array}$ & $\begin{array}{r}8.0 \\
48.0 \\
124.0 \\
70.0 \\
\\
<10.0\end{array}$ & $\begin{array}{r}12,600 \\
12,350 \\
16,900 \\
18,700 \\
14,050 \\
13,200 \\
8,800\end{array}$ & $\begin{array}{r}8,580 \\
8,590 \\
13,950 \\
16,200 \\
12,300 \\
11,100 \\
4,950\end{array}$ & $\begin{array}{l}3,230 \\
2,940 \\
1,900 \\
1,980 \\
1,460 \\
1,580 \\
3,130\end{array}$ & $\begin{array}{r}269 \\
288 \\
294 \\
19 \\
37 \\
19 \\
225\end{array}$ \\
\hline A. D. & $\begin{array}{r}0 \\
1 \\
4 \\
8 \\
24\end{array}$ & $\begin{array}{r}8.4 \\
107.2 \\
61.2 \\
5.4\end{array}$ & $\begin{array}{r}5,080 \\
6,650 \\
10,450 \\
9,850 \\
6,250\end{array}$ & $\begin{array}{l}2,733 \\
4,043 \\
9,446 \\
7,900 \\
3,450\end{array}$ & $\begin{array}{r}2,022 \\
2,141 \\
836 \\
1,694 \\
2,350\end{array}$ & $\begin{array}{r}88 \\
125 \\
25 \\
6 \\
76\end{array}$ \\
\hline O. H. & $\begin{array}{r}0 \\
1 \\
4 \\
8 \\
24\end{array}$ & $\begin{array}{r}18.2 \\
63.2 \\
29.3 \\
7.7 \\
6.2\end{array}$ & $\begin{array}{r}7,100 \\
7,600 \\
19,050 \\
13,600 \\
7,300\end{array}$ & $\begin{array}{r}4,075 \\
4,530 \\
17,640 \\
11,750 \\
3,781\end{array}$ & $\begin{array}{r}2,144 \\
2,446 \\
838 \\
1,442 \\
2,964\end{array}$ & $\begin{array}{r}325 \\
300 \\
6 \\
0 \\
263\end{array}$ \\
\hline D. T. & $\begin{array}{r}0 \\
1 \\
4 \\
8 \\
24\end{array}$ & \begin{tabular}{r|}
8.0 \\
80.2 \\
60.1 \\
13.7 \\
11.4
\end{tabular} & $\begin{array}{r}8,950 \\
7,650 \\
8,900 \\
12,500 \\
7,700\end{array}$ & $\begin{array}{r}4,887 \\
5,110 \\
7,191 \\
11,325 \\
4,004\end{array}$ & $\begin{array}{r}3,204 \\
1,943 \\
1,549 \\
925 \\
3,234\end{array}$ & $\begin{array}{r}263 \\
138 \\
25 \\
6 \\
263\end{array}$ \\
\hline J. E. & $\begin{array}{r}0 \\
1 \\
4 \\
8 \\
24\end{array}$ & $\begin{array}{r}10.7 \\
63.6 \\
50.0 \\
\\
2.4\end{array}$ & $\begin{array}{r}5,000 \\
5,350 \\
5,900 \\
10,000 \\
8,400\end{array}$ & $\begin{array}{l}2,830 \\
2,857 \\
4,838 \\
9,020 \\
3,914\end{array}$ & $\begin{array}{r}1,730 \\
1,937 \\
896 \\
780 \\
3,814\end{array}$ & $\begin{array}{r}181 \\
194 \\
19 \\
0 \\
238\end{array}$ \\
\hline J. B. & $\begin{array}{r}0 \\
1 \\
4 \\
8 \\
24\end{array}$ & $\begin{array}{r}11.0 \\
87.0 \\
58.0 \\
9.0 \\
15.0\end{array}$ & $\begin{array}{l}4,400 \\
4,900 \\
6,700 \\
7,950 \\
5,550\end{array}$ & $\begin{array}{l}2,692 \\
3,386 \\
6,372 \\
5,624 \\
2,950\end{array}$ & $\begin{array}{l}2,659 \\
2,789 \\
1,057 \\
3,572 \\
2,549\end{array}$ & $\begin{array}{r}100 \\
113 \\
13 \\
6 \\
138\end{array}$ \\
\hline T. 0. & $\begin{array}{r}0 \\
1 \\
4 \\
8 \\
24\end{array}$ & $\begin{array}{l}11.0 \\
98.0 \\
50.0 \\
21.3 \\
20.0\end{array}$ & $\begin{array}{r}6,160 \\
7,400 \\
18,350 \\
12,675 \\
13,400\end{array}$ & $\begin{array}{r}4,151 \\
5,239 \\
16,698 \\
10,977 \\
8,576\end{array}$ & $\begin{array}{l}1,602 \\
1,746 \\
1,431 \\
1,369 \\
4,100\end{array}$ & $\begin{array}{r}150 \\
100 \\
19 \\
6 \\
106\end{array}$ \\
\hline
\end{tabular}




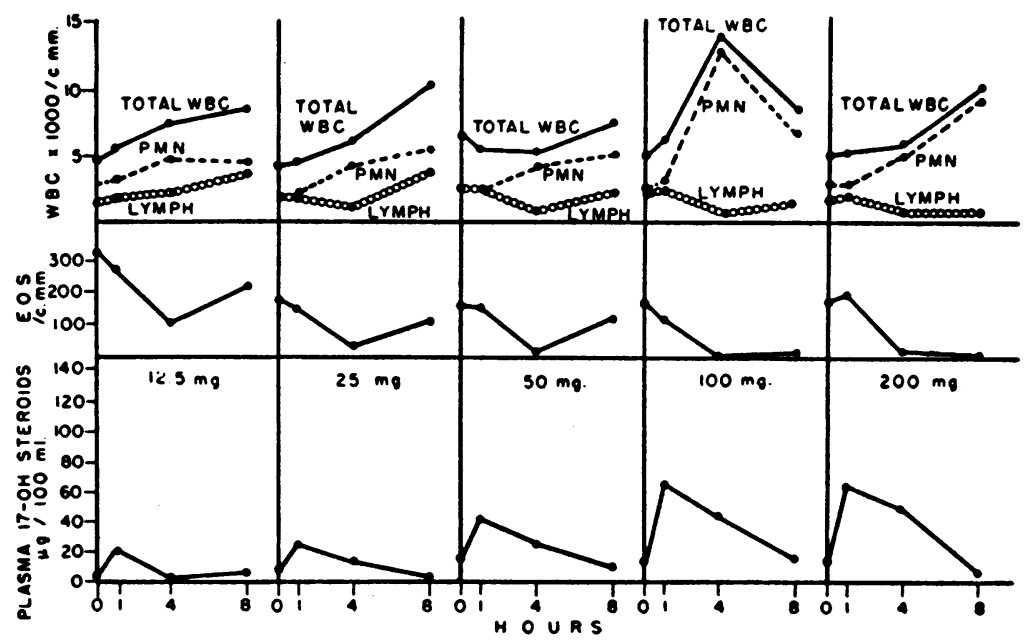

Fig. 2. The Effect of Varying Doses of Compound F Acetate Given Orally to a Single Subject

The effects of $200 \mathrm{mgm}$. of cortisone acetate given orally to five subjects are tabulated in Table III. The effects were similar to those seen following compound $F$ acetate given orally, but were less marked. The rise in steroid levels was in most cases not so great and was less prolonged; the same is true of the changes in leukocytes. In

TABLE II

Administration of 200 mgm. 17-hydroxycorticosterone (Compound $F$ ) acetate $I$. $M$.

\begin{tabular}{|c|c|c|c|c|c|c|}
\hline \multirow{2}{*}{$\underset{\text { ject }}{\text { Sub- }}$} & \multirow{2}{*}{$\underset{\substack{\text { in } \\
\text { hours }}}{\text { Time }}$} & \multirow{2}{*}{$\begin{array}{c}17-\mathrm{OH} \\
\text { steroid } \\
\text { levels, } \\
\gamma / 100 \mathrm{ml} . \\
\text { of plasma }\end{array}$} & \multirow{2}{*}{ WBC } & PMN & Lymph & \multirow{2}{*}{ EOS } \\
\hline & & & & \multicolumn{2}{|c|}{$\begin{array}{c}\text { Absolute number } \\
\text { per mm. }\end{array}$} & \\
\hline T. 0. & $\begin{array}{r}0 \\
1 \\
4 \\
8 \\
24\end{array}$ & $\begin{array}{l}19 \\
19.2 \\
8.4 \\
6 \\
4\end{array}$ & $\begin{array}{r}7,850 \\
5,900 \\
8,250 \\
10,900 \\
7,350\end{array}$ & $\begin{array}{l}4,773 \\
3,741 \\
5,659 \\
8,349 \\
4,293\end{array}$ & $\begin{array}{l}2,685 \\
1,841 \\
2,145 \\
2,180 \\
2,675\end{array}$ & $\begin{array}{r}113 \\
154 \\
156 \\
175 \\
81\end{array}$ \\
\hline M. C. & $\begin{array}{r}0 \\
1 \\
4 \\
8 \\
24\end{array}$ & $\begin{array}{l}4.6 \\
0 \\
8.8 \\
8.0 \\
1.0\end{array}$ & $\begin{array}{l}13,150 \\
13,200 \\
12,900 \\
11,750 \\
16,600\end{array}$ & $\begin{array}{r}7,601 \\
8,791 \\
8,514 \\
7,755 \\
11,919\end{array}$ & $\begin{array}{l}4,576 \\
3,379 \\
3,638 \\
3,219 \\
3,386\end{array}$ & $\begin{array}{l}294 \\
356 \\
400 \\
250 \\
331\end{array}$ \\
\hline J. B. & $\begin{array}{r}0 \\
1 \\
4 \\
8 \\
24\end{array}$ & $\begin{array}{l}19 \\
22.6 \\
14.4 \\
12 \\
16.8\end{array}$ & $\begin{array}{l}\mathbf{5}, 500 \\
\mathbf{5}, 900 \\
7,500 \\
8,650 \\
5,050\end{array}$ & $\begin{array}{l}2,387 \\
2,773 \\
3,960 \\
5,916 \\
2,545\end{array}$ & $\begin{array}{l}2,640 \\
2,669 \\
3,090 \\
2,301 \\
2,122\end{array}$ & $\begin{array}{r}106 \\
81 \\
81 \\
75 \\
125\end{array}$ \\
\hline A. D. & 0 & $\begin{array}{r}8.0 \\
10.2\end{array}$ & 6,400 & 4,250 & 1,920 & 100 \\
\hline & $\begin{array}{l}2 \\
1 \\
2 \\
4 \\
6 \\
8\end{array}$ & $\begin{array}{l}4.2 \\
5.4\end{array}$ & $\begin{array}{l}6,700 \\
7,800 \\
8,350 \\
8,550 \\
9,150\end{array}$ & $\begin{array}{l}4,680 \\
5,410 \\
5,650 \\
6,550 \\
6,860\end{array}$ & $\begin{array}{l}1,860 \\
1,860 \\
1,950 \\
1,408 \\
1,755\end{array}$ & $\begin{array}{r}69 \\
150 \\
75 \\
100 \\
50\end{array}$ \\
\hline
\end{tabular}

Table IV are shown data on four subjects given cortisone acetate intramuscularly. The results are less consistent than those shown previously. In one subject (C. C.), there was a marked rise

TABLE III

Administration of $200 \mathrm{mgm}$. 17-hydroxy-11-dehydrocorticosterone (Compound E) acetate orally

\begin{tabular}{|c|c|c|c|c|c|c|}
\hline \multirow{2}{*}{ Sub- } & \multirow{2}{*}{$\underset{\substack{\text { in } \\
\text { hours }}}{\text { Time }}$} & \multirow{2}{*}{$\begin{array}{c}17-0 H \\
\text { steroid } \\
\text { levels, } \\
\gamma / 100 \mathrm{ml} . \\
\text { of plasma }\end{array}$} & \multirow{2}{*}{ WBC } & $\mathbf{P M N}$ & Lymph & \multirow{2}{*}{ EOS } \\
\hline & & & & \multicolumn{2}{|c|}{$\begin{array}{l}\text { Absolute number } \\
\text { per mm. }\end{array}$} & \\
\hline O. H. & $\begin{array}{r}0 \\
1 \\
4 \\
8 \\
24\end{array}$ & \begin{tabular}{r|}
14 \\
25.2 \\
11.7 \\
9.7 \\
8.0
\end{tabular} & $\begin{array}{r}8,700 \\
9,500 \\
10,100 \\
10,200 \\
8,800\end{array}$ & $\begin{array}{l}4,768 \\
5,833 \\
8,040 \\
6,936 \\
4,453\end{array}$ & $\begin{array}{l}2,871 \\
2,907 \\
1,575 \\
2,754 \\
3,802\end{array}$ & $\begin{array}{r}344 \\
288 \\
25 \\
90 \\
281\end{array}$ \\
\hline D. T. & $\begin{array}{r}0 \\
1 \\
4 \\
8 \\
24\end{array}$ & $\begin{array}{r}7.8 \\
46.0 \\
20.0 \\
4.0 \\
6.0\end{array}$ & $\begin{array}{l}6,200 \\
6,600 \\
6,950 \\
9,000 \\
6,800\end{array}$ & $\begin{array}{l}3,174 \\
3,986 \\
5,463 \\
5,580 \\
2,679\end{array}$ & $\begin{array}{l}2,554 \\
1,848 \\
1,251 \\
2,898 \\
3,754\end{array}$ & $\begin{array}{r}125 \\
225 \\
25 \\
69 \\
194\end{array}$ \\
\hline C. C. & $\begin{array}{r}0 \\
1 \\
4 \\
8 \\
24\end{array}$ & $\begin{array}{r}3.0 \\
124.0 \\
21.0 \\
7.0 \\
4.0\end{array}$ & $\begin{array}{r}8,500 \\
9,100 \\
12,600 \\
12,400 \\
8,450\end{array}$ & $\begin{array}{r}4,641 \\
5,187 \\
10,483 \\
9,796 \\
4,326\end{array}$ & $\begin{array}{l}3,043 \\
3,130 \\
1,789 \\
2,306 \\
3,566\end{array}$ & $\begin{array}{r}238 \\
275 \\
13 \\
56 \\
269\end{array}$ \\
\hline C. F. & $\begin{array}{r}0 \\
1 \\
4 \\
8 \\
24\end{array}$ & $\begin{array}{r}11.0 \\
65.0 \\
16.0 \\
5.0 \\
10.0\end{array}$ & $\begin{array}{r}10,800 \\
11,000 \\
9,500 \\
7,850 \\
7,800\end{array}$ & $\begin{array}{l}6,523 \\
6,490 \\
7,163 \\
4,569 \\
3,479\end{array}$ & $\begin{array}{l}3,370 \\
3,564 \\
1,995 \\
2,747 \\
3,526\end{array}$ & $\begin{array}{r}394 \\
369 \\
62 \\
131 \\
356\end{array}$ \\
\hline T. 0 . & $\begin{array}{r}0 \\
1 \\
4 \\
8 \\
24\end{array}$ & $\begin{array}{r}9.6 \\
50.0 \\
33.0 \\
1.3 \\
4.0\end{array}$ & $\begin{array}{r}7,500 \\
6,850 \\
6,050 \\
12,750 \\
9,000\end{array}$ & $\begin{array}{r}4,440 \\
4,370 \\
4,985 \\
10,404 \\
5,760\end{array}$ & $\begin{array}{r}2,775 \\
2,206 \\
920 \\
1,912 \\
2,664\end{array}$ & $\begin{array}{r}81 \\
100 \\
38 \\
44 \\
138\end{array}$ \\
\hline
\end{tabular}


TABLE IV

Administration of $200 \mathrm{mgm}$. of 17-hydroxy-11-dehydrocorticosterone (Compound $E$ ) acetate I. M.

\begin{tabular}{|c|c|c|c|c|c|c|}
\hline \multirow{2}{*}{ Sub- $_{\text {ject }}$} & \multirow{2}{*}{$\underset{\substack{\text { in } \\
\text { hours }}}{\text { Time }}$} & \multirow{2}{*}{$\begin{array}{c}\text { 17-OH } \\
\text { steroid } \\
\text { levels, } \\
\gamma / 100 \mathrm{ml} . \\
\text { of plasma }\end{array}$} & \multirow{2}{*}{ WBC } & PMN & Lymph & \multirow{2}{*}{ EOS } \\
\hline & & & & \multicolumn{2}{|c|}{$\begin{array}{l}\text { Absolute number } \\
\text { per mm. }\end{array}$} & \\
\hline C. C. & $\begin{array}{r}0 \\
1 \\
4 \\
8 \\
24\end{array}$ & $\begin{array}{l}16.8 \\
59.0 \\
31.0 \\
31.0 \\
29.0\end{array}$ & $\begin{array}{r}6,550 \\
8,450 \\
7,400 \\
16,800 \\
15,400\end{array}$ & $\begin{array}{r}3,733 \\
4,343 \\
5,105 \\
14,112 \\
12,166\end{array}$ & $\begin{array}{l}2,306 \\
3,211 \\
1,806 \\
1,949 \\
2,279\end{array}$ & $\begin{array}{r}238 \\
300 \\
113 \\
75 \\
69\end{array}$ \\
\hline C. F. & $\begin{array}{r}0 \\
1 \\
4 \\
8 \\
24 \\
53\end{array}$ & $\begin{array}{l}16 \\
16 \\
15 \\
14 \\
20 \\
13\end{array}$ & $\begin{array}{r}5,800 \\
7,200 \\
9,650 \\
11,400 \\
6,600 \\
7,400\end{array}$ & $\begin{array}{l}2,657 \\
3,715 \\
5,616 \\
7,136 \\
3,168 \\
3,480\end{array}$ & $\begin{array}{l}2,668 \\
2,866 \\
3,203 \\
3,466 \\
2,600 \\
2,796\end{array}$ & $\begin{array}{l}381 \\
250 \\
288 \\
394 \\
413 \\
400\end{array}$ \\
\hline T. 0. & $\begin{array}{r}0 \\
1 \\
4 \\
8 \\
24\end{array}$ & $\begin{array}{l}17 \\
23 \\
21 \\
18 \\
10\end{array}$ & $\begin{array}{r}9,900 \\
8,160 \\
10,050 \\
20,500 \\
14,950\end{array}$ & $\begin{array}{r}6,276 \\
5,615 \\
7,919 \\
17,220 \\
12,528\end{array}$ & $\begin{array}{l}2,990 \\
1,909 \\
1,729 \\
2,009 \\
1,973\end{array}$ & $\begin{array}{r}134 \\
134 \\
75 \\
109 \\
150\end{array}$ \\
\hline J. E. & $\begin{array}{r}0 \\
1 \\
4 \\
8 \\
24\end{array}$ & $\begin{array}{l}13.2 \\
18.0 \\
12.0 \\
28.0 \\
14.4\end{array}$ & $\begin{array}{r}6,120 \\
5,700 \\
4,950 \\
14,550 \\
9,180\end{array}$ & $\begin{array}{r}3,427 \\
3,146 \\
3,385 \\
12,455 \\
6,940\end{array}$ & $\begin{array}{l}2,069 \\
2,143 \\
1,228 \\
1,484 \\
1,671\end{array}$ & $\begin{array}{r}125 \\
106 \\
47 \\
32 \\
91\end{array}$ \\
\hline
\end{tabular}

in steroids with a moderate drop in lymphocytes and eosinophils, and definite neutrophilia. These effects persisted, however, and were still present 24 hours after administration of the compound. In another subject (C. F.) no significant change

TABLE V

Administration of 17-hydroxycorticosterone (Compound F) as the free alcohol, $100 \mathrm{mgm}$.

\begin{tabular}{|c|c|c|c|c|c|c|c|}
\hline \multirow{3}{*}{$\begin{array}{c}\begin{array}{c}\text { Sub- } \\
\text { ject }\end{array} \\
\text { J. B. }\end{array}$} & \multirow{3}{*}{$\begin{array}{l}\begin{array}{c}\text { Mode of } \\
\text { admin. }\end{array} \\
100 \text { mgm. } \\
\text { I. M. in } \\
33 \% \\
\text { ethanol }\end{array}$} & \multirow{3}{*}{$\begin{array}{c}\begin{array}{c}\text { Time } \\
\text { in } \\
\text { hours }\end{array} \\
0 \\
1 \\
4 \\
8 \\
24\end{array}$} & \multirow{3}{*}{$\begin{array}{c}17-\mathrm{OH} \\
\text { steroid } \\
\text { levels, } \\
\gamma / 100 \mathrm{ml} \text {. } \\
\text { of plasma } \\
16 \\
29 \\
13 \\
8 \\
11\end{array}$} & \multirow{3}{*}{\begin{tabular}{|l|} 
\\
WBC \\
4,400 \\
4,900 \\
6,700 \\
7,950 \\
5,550
\end{tabular}} & PMN & Lymph & \multirow{3}{*}{$\begin{array}{r}\text { EOS } \\
\\
113 \\
91 \\
31 \\
43 \\
119\end{array}$} \\
\hline & & & & & \multicolumn{2}{|c|}{$\begin{array}{l}\text { Absolute } \\
\text { number } \\
\text { per mm. }\end{array}$} & \\
\hline & & & & & $\begin{array}{l}1,892 \\
2,293 \\
3,953 \\
4,865 \\
2,620\end{array}$ & $\begin{array}{l}2,112 \\
2,273 \\
2,386 \\
2,623 \\
2,542\end{array}$ & \\
\hline O. H. & $\begin{array}{l}100 \text { mgm. } \\
\text { I. M. in } \\
33 \% \\
\text { ethanol }\end{array}$ & $\begin{array}{r}0 \\
1 \\
4 \\
8 \\
24\end{array}$ & $\begin{array}{r}18.0 \\
49.0 \\
21.0 \\
6.0 \\
2.0\end{array}$ & $\begin{array}{r}7,800 \\
7,200 \\
16,400 \\
13,670 \\
8,280\end{array}$ & $\begin{array}{r}3,947 \\
4,737 \\
15,055 \\
11,784 \\
4,951\end{array}$ & $\begin{array}{l}2,902 \\
1,872 \\
1,016 \\
1,449 \\
2,600\end{array}$ & $\begin{array}{r}300 \\
228 \\
12 \\
9 \\
241\end{array}$ \\
\hline J. B. & $\begin{array}{l}100 \text { mgm. } \\
\text { orally }\end{array}$ & $\begin{array}{r}0 \\
1 \\
4 \\
8 \\
24\end{array}$ & $\begin{array}{r}3.0 \\
30.0 \\
34.0 \\
6.6 \\
10.6\end{array}$ & $\begin{array}{l}6,400 \\
5,550 \\
8,700 \\
8,100 \\
6,400\end{array}$ & $\begin{array}{l}2,650 \\
2,442 \\
6,177 \\
5,233 \\
3,213\end{array}$ & $\begin{array}{l}3,366 \\
2,620 \\
2,123 \\
2,268 \\
2,777\end{array}$ & $\begin{array}{r}75 \\
81 \\
6 \\
31 \\
81\end{array}$ \\
\hline O. H. & $\begin{array}{l}100 \text { mgm. } \\
\text { orally }\end{array}$ & $\begin{array}{r}0 \\
1 \\
4 \\
8 \\
24\end{array}$ & $\begin{array}{r}5.0 \\
70.0 \\
22.5 \\
2.5 \\
9.0\end{array}$ & $\begin{array}{r}8,150 \\
9,350 \\
20,100 \\
13,950 \\
7,250\end{array}$ & $\begin{array}{r}4,304 \\
4,937 \\
17,889 \\
11,272 \\
3,814\end{array}$ & $\begin{array}{l}2,950 \\
3,310 \\
1,688 \\
2,204 \\
2,624\end{array}$ & $\begin{array}{r}300 \\
283 \\
13 \\
44 \\
256\end{array}$ \\
\hline
\end{tabular}

TABLE VI

Oral administration of 17-hydroxy-11-dehydrocorticosterone (Compound $E)$ as the free alcohol orally $(200 \mathrm{mgm}$.

\begin{tabular}{|c|c|c|c|c|c|c|}
\hline \multirow{2}{*}{$\underbrace{\text { Sub- }}_{\text {ject }}$} & \multirow{2}{*}{$\begin{array}{c}\text { Time } \\
\text { in } \\
\text { hours }\end{array}$} & \multirow{2}{*}{$\begin{array}{c}17-\mathrm{OH} \\
\text { steroid } \\
\text { levels, } \\
\gamma / 100 \mathrm{ml} . \\
\text { of plasma }\end{array}$} & \multirow{2}{*}{ WBC } & PMN & Lymph & \multirow{2}{*}{ EOS } \\
\hline & & & & \multicolumn{2}{|c|}{$\begin{array}{l}\text { Absolute number } \\
\text { per mm. }\end{array}$} & \\
\hline C. C. & $\begin{array}{r}0 \\
1 \\
4 \\
8 \\
24\end{array}$ & $\begin{array}{r}8.0 \\
70.0 \\
14.0 \\
0.6 \\
7.0\end{array}$ & $\begin{array}{r}7,850 \\
9,000 \\
6,600 \\
10,350 \\
10,900\end{array}$ & $\begin{array}{l}4,698 \\
4,688 \\
4,963 \\
6,541 \\
6,300\end{array}$ & $\begin{array}{l}2,685 \\
3,510 \\
1,439 \\
3,374 \\
4,164\end{array}$ & $\begin{array}{r}294 \\
313 \\
38 \\
69 \\
450\end{array}$ \\
\hline J. C. & $\begin{array}{r}0 \\
1 \\
4 \\
8 \\
24\end{array}$ & $\begin{array}{r}13.0 \\
58.0 \\
41.0 \\
7.5 \\
2.5\end{array}$ & $\begin{array}{r}11,475 \\
9,550 \\
9,500 \\
12,300 \\
13,650\end{array}$ & $\begin{array}{l}6,174 \\
5,635 \\
7,372 \\
8,561 \\
6,607\end{array}$ & $\begin{array}{l}4,360 \\
3,476 \\
1,691 \\
3,247 \\
5,979\end{array}$ & $\begin{array}{r}213 \\
213 \\
50 \\
25 \\
225\end{array}$ \\
\hline C. F. & $\begin{array}{r}0 \\
1 \\
4 \\
8 \\
24\end{array}$ & $\begin{array}{r}5.4 \\
35.0 \\
41.8 \\
17.2 \\
14.5\end{array}$ & $\begin{array}{r}9,250 \\
8,050 \\
7,200 \\
10,650 \\
9,150\end{array}$ & $\begin{array}{l}4,126 \\
3,848 \\
4,939 \\
7,881 \\
5,398\end{array}$ & $\begin{array}{l}3,811 \\
3,188 \\
1,814 \\
2,279 \\
2,965\end{array}$ & $\begin{array}{r}519 \\
500 \\
175 \\
69 \\
325\end{array}$ \\
\hline
\end{tabular}

was seen in either cells or steroids. In the other two subjects the changes were not marked, and in one (J. E.) were delayed.

Table V shows the effects of compound $\mathrm{F}$ given orally and intramuscularly (in ethanol solution) in the form of the free alcohol in a dose of 100 mgm. It may be seen that a rise in steroids with typical changes in cells occurred in each case by either route of administration with the same time relationships as are noted following oral $\mathrm{F}$ acetate ingestion. Oral administration of cortisone resulted in similar changes, as shown in Table VI.

TABLE VII

Administration of $200 \mathrm{mgm}$. 17-hydroxy-11-dehydrocorticosterone (Compound $E$ ) as the free alcohol, I. $M$.

\begin{tabular}{|c|c|c|c|c|c|c|c|}
\hline \multirow{2}{*}{$\underset{\text { ject }}{\text { Sub- }}$} & \multirow{2}{*}{$\begin{array}{l}\text { Mode of } \\
\text { admin. }\end{array}$} & \multirow{2}{*}{$\begin{array}{c}\text { Time } \\
\text { in } \\
\text { hours }\end{array}$} & \multirow{2}{*}{$\begin{array}{c}17-\mathrm{OH} \\
\text { steroid } \\
\text { levels, } \\
\gamma / 100 \mathrm{ml} \text {. } \\
\text { of plasma }\end{array}$} & \multirow[b]{2}{*}{ WBC } & PMN & Lymph & \multirow[b]{2}{*}{ EOS } \\
\hline & & & & & \multicolumn{2}{|c|}{$\begin{array}{c}\text { Absolute } \\
\text { number } \\
\text { per mm. }\end{array}$} & \\
\hline C. C. & $\begin{array}{l}\text { Suspended } \\
\text { in saline, } \\
\text { I. M. }\end{array}$ & $\begin{array}{r}\mathbf{0} \\
1 \\
\mathbf{4} \\
\mathbf{8} \\
\mathbf{2 4}\end{array}$ & $\begin{array}{r}11.2 \\
5.6 \\
1.4 \\
5.6 \\
4.2\end{array}$ & $\begin{array}{r}8,200 \\
7,650 \\
8,650 \\
10,600 \\
11,250\end{array}$ & $\begin{array}{l}4,592 \\
4,192 \\
5,173 \\
6,508 \\
6,862\end{array}$ & $\begin{array}{l}2,903 \\
2,693 \\
2,612 \\
3,456 \\
3,623\end{array}$ & $\begin{array}{l}213 \\
206 \\
169 \\
175 \\
214\end{array}$ \\
\hline C. F. & $\begin{array}{l}\text { Dissolved } \\
\text { in } 25 \% \\
\text { ethanol, } \\
\text { I. } M \text {. }\end{array}$ & $\begin{array}{r}0 \\
1 \\
4 \\
8 \\
24\end{array}$ & $\begin{array}{r}11.0 \\
38.0 \\
26.0 \\
21.0 \\
2.5\end{array}$ & $\begin{array}{r}10,650 \\
11,700 \\
9,550 \\
14,800 \\
12,200\end{array}$ & $\begin{array}{r}6,411 \\
8,237 \\
7,736 \\
11,574 \\
7,491\end{array}$ & $\begin{array}{l}3,365 \\
2,481 \\
1,452 \\
2,723 \\
3,684\end{array}$ & $\begin{array}{r}450 \\
406 \\
138 \\
75 \\
238\end{array}$ \\
\hline J. C. & $\begin{array}{l}\text { Dissolved } \\
\text { in } 33 \% \\
\text { ethanol, } \\
\text { I. } \mathbf{M} \text {. }\end{array}$ & $\begin{array}{r}0 \\
1 \\
4 \\
8 \\
24\end{array}$ & $\begin{array}{r}10.5 \\
46.0 \\
21.0 \\
21.6 \\
6.6\end{array}$ & $\begin{array}{r}9,380 \\
9,050 \\
11,400 \\
16,125 \\
14,950\end{array}$ & $\begin{array}{r}4,821 \\
5,321 \\
8,824 \\
10,933 \\
10,226\end{array}$ & $\begin{array}{l}3,883 \\
3,168 \\
2,165 \\
4,676 \\
3,947\end{array}$ & $\begin{array}{r}235 \\
238 \\
62 \\
59 \\
244\end{array}$ \\
\hline
\end{tabular}


TABLE VIII

Administration of 17-hydroxy-11-desoxycorticosterone (Compound $S$ ) as the free alcohol or acetate

\begin{tabular}{|c|c|c|c|c|c|c|c|}
\hline \multirow{2}{*}{ Subject } & \multirow{2}{*}{$\begin{array}{l}\text { Dose and form of steroid } \\
\text { and route of admin. }\end{array}$} & \multirow{2}{*}{$\underset{\text { hours }}{\text { Time in }}$} & \multirow{2}{*}{$\begin{array}{l}17-\mathrm{OH} \\
\text { steroid levels, } \\
\gamma / 100 \mathrm{ml} . \\
\text { of plasma }\end{array}$} & \multirow{2}{*}{ WBC } & PMN & Lymph & \multirow{2}{*}{ EOS } \\
\hline & & & & & \multicolumn{2}{|c|}{ Absolute number per $\mathrm{mm} .^{\mathrm{a}}$} & \\
\hline O. H. & $\begin{array}{l}\text { 17-hydroxy-11-desoxy } \\
\text { corticosterone acetate } \\
\text { (Compound S acetate) } \\
200 \text { mgm. orally }\end{array}$ & $\begin{array}{r}0 \\
1 \\
4 \\
8 \\
24\end{array}$ & $\begin{array}{r}18 \\
11 \\
9 \\
5 \\
11\end{array}$ & $\begin{array}{r}9,550 \\
7,900 \\
7,900 \\
10,750 \\
6,700\end{array}$ & $\begin{array}{l}4,737 \\
3,824 \\
4,172 \\
5,934 \\
3,510\end{array}$ & $\begin{array}{l}3,839 \\
3,144 \\
2,924 \\
3,655 \\
2,640\end{array}$ & $\begin{array}{l}244 \\
256 \\
288 \\
238 \\
306\end{array}$ \\
\hline O. H. & $\begin{array}{l}\text { As above. Given I. M. } \\
\text { in } 10 \% \text { ethanol }\end{array}$ & $\begin{array}{r}0 \\
1 \\
4 \\
8 \\
24\end{array}$ & $\begin{array}{r}6 \\
15 \\
16 \\
10 \\
5\end{array}$ & $\begin{array}{r}8,400 \\
7,350 \\
9,000 \\
10,000 \\
7,450\end{array}$ & $\begin{array}{l}4,570 \\
4,292 \\
5,634 \\
5,880 \\
4,232\end{array}$ & $\begin{array}{l}2,688 \\
2,264 \\
2,826 \\
3,220 \\
2,502\end{array}$ & $\begin{array}{l}188 \\
238 \\
225 \\
200 \\
188\end{array}$ \\
\hline J. C. & $\begin{array}{l}\text { 17-hydroxy-11-desoxy- } \\
\text { corticosterone (free } \\
\text { alcohol), } 200 \mathrm{mgm} \text {. } \\
\text { orally }\end{array}$ & $\begin{array}{r}0 \\
1 \\
4 \\
8 \\
24\end{array}$ & $\begin{array}{l}15 \\
14 \\
14 \\
11 \\
22\end{array}$ & $\begin{array}{r}9,100 \\
9.100 \\
11,750 \\
13,250 \\
11,200\end{array}$ & $\begin{array}{l}5,041 \\
4,787 \\
6,627 \\
7,253 \\
6,294\end{array}$ & $\begin{array}{l}3,531 \\
3,786 \\
4,230 \\
5,088 \\
4,055\end{array}$ & $\begin{array}{l}144 \\
144 \\
200 \\
400 \\
238\end{array}$ \\
\hline J. C. & $\begin{array}{l}\text { Free alcohol of } \\
\text { Compound S } \\
\text { I. M., } 200 \mathrm{mgm} . \text { in } \\
25 \% \text { ethanol }\end{array}$ & $\begin{array}{r}0 \\
1 \\
4 \\
8 \\
24\end{array}$ & $\begin{array}{l}0.3 \\
9.6 \\
1.3 \\
1.3 \\
4.0\end{array}$ & $\begin{array}{l}10,700 \\
10,950 \\
12,050 \\
15,900 \\
15,250\end{array}$ & $\begin{array}{r}\mathbf{5 , 7 5 6} \\
\mathbf{6 , 7 4 5} \\
\mathbf{7 , 2 0 6} \\
\mathbf{7 , 9 0 5} \\
10,858\end{array}$ & $\begin{array}{l}4,045 \\
3,504 \\
3,977 \\
7,028 \\
3,325\end{array}$ & $\begin{array}{l}256 \\
200 \\
181 \\
325 \\
175\end{array}$ \\
\hline
\end{tabular}

Intramuscular administration of free cortisone produced no changes when given suspended in saline, but when given in ethanol solution, typical responses occurred, as shown in Table VII.

Compound S, given as the acetate or free alcohol, either intramuscularly or orally, failed to affect significantly the blood steroid levels or the circulating leukocytes, as shown in Table VIII.

Table IX shows the diurnal variations in ster-

TABLE IX

Diurnal variation

\begin{tabular}{|c|c|c|c|c|c|c|}
\hline \multirow{2}{*}{$\underset{\text { ject }}{\text { Sub- }}$} & \multirow{2}{*}{$\begin{array}{c}\text { Time in } \\
\text { hours }\end{array}$} & \multirow{2}{*}{$\begin{array}{c}17-\mathrm{OH} \\
\text { steroid } \\
\text { levels, } \\
\gamma / 100 \mathrm{ml} . \\
\text { of plasma }\end{array}$} & \multirow[b]{2}{*}{ WBC } & PMN & Lymph & \multirow[b]{2}{*}{ EOS } \\
\hline & & & & \multicolumn{2}{|c|}{$\begin{array}{c}\text { Absolute } \\
\text { number } \\
\text { per mm.? }\end{array}$} & \\
\hline O. H. & $\begin{array}{l}0 \text { (8 a.m.) } \\
1 \\
4 \\
8\end{array}$ & $\begin{array}{r}10.0 \\
12.0 \\
11.0 \\
3.0\end{array}$ & $\begin{array}{r}8,250 \\
9,700 \\
8,500 \\
10,700\end{array}$ & $\begin{array}{l}4,901 \\
5,937 \\
5,525 \\
6,163\end{array}$ & $\begin{array}{l}2,656 \\
2,968 \\
2,397 \\
3,745\end{array}$ & $\begin{array}{l}150 \\
250 \\
331 \\
100\end{array}$ \\
\hline D. T. & $\begin{array}{l}0 \text { (8 a.m.) } \\
1 \\
4 \\
8\end{array}$ & $\begin{array}{l}9.1 \\
7.5 \\
9.2 \\
8.1\end{array}$ & $\begin{array}{l}7,100 \\
7,900 \\
9,800 \\
9,400\end{array}$ & $\begin{array}{l}4,033 \\
5,087 \\
5,762 \\
5,189\end{array}$ & $\begin{array}{l}2,215 \\
2,244 \\
2,940 \\
3,272\end{array}$ & $\begin{array}{l}225 \\
175 \\
231 \\
269\end{array}$ \\
\hline O. H. & $\begin{array}{l}0 \text { (8 a.m.) } \\
1 \\
4 \\
8\end{array}$ & & $\begin{array}{r}7,450 \\
7,350 \\
9,200 \\
11,200\end{array}$ & $\begin{array}{l}4,038 \\
3,014 \\
5,484 \\
6,854\end{array}$ & $\begin{array}{l}2,563 \\
3,836 \\
2,889 \\
3,517\end{array}$ & $\begin{array}{l}256 \\
156 \\
206 \\
206\end{array}$ \\
\hline
\end{tabular}

oids and cells of two subjects, one of whom was studied on two separate days. Any changes which can be attributed to the administered hormone must fall outside this range.

\section{DISCUSSION}

From these data it is apparent that following the oral administration of compound $F$ or cortisone, either as the acetate ester or the free alcohol, there results a prompt rise in plasma 17hydroxycorticosteroid levels, reaching a peak at approximately one hour and gradually falling thereafter. Although the highest levels appear to occur at one hour, no quantitative significance can be attached to the actual value since it is undoubtedly changing rapidly at this time. The levels at four hours are probably changing less rapidly and may be more indicative of the degree of change in steroid level which has occurred. This is well illustrated by the progressively rising four-hour levels in the individual who received various doses of compound $\mathrm{F}$ acetate. By eight hours in every case the steroids had returned to control levels, and in many instances they were below these values, suggesting that adrenal activity may have been suppressed by the previous phase of artifi- 
cially increased steroid concentration. In some cases this was still apparent at 24 hours.

In contrast to the change in steroid levels, the changes in circulating leukocytes were rarely seen at one hour, and were usually maximal at four to eight hours after oral administration of the compounds. In many instances, when the maximal leukocyte alteration occurred at eight hours, the four-hour steroid level was relatively higher, suggesting that the maximum rise in steroids was later than one hour after administration of the steroid. Thus the leukocyte changes appear to lag behind the steroid change by about three to six hours. The occurrence of this lag is of considerable interest in relation to the mechanism of the cellular changes, but the reason for the delay is unknown at present.

Decreases in the average number of both eosinophils and lymphocytes were seen in every case when a significant elevation in steroid levels occurred. The alterations in these two cell types were, in general, closely parallel. At times, the numbers of each were higher at 24 hours than at any time previously. This eosinophilia and lymphocytosis may reflect a period of decreased adrenal activity as previously mentioned, or may be the result of increased cell production following their destruction (1). Changes in lymphocytes and eosinophils were well correlated with dosage in the individual who received graded doses of compound F acetate. However, the degree of cellular change from individual to individual could not be predicted from the steroid level. Thus, one individual may exhibit changes in the plasma steroid level of considerable magnitude and show only modest changes in the leukocytes, whereas another who may manifest a minimal elevation in the steroid level may show profound changes in the leukocytes.

Neutrophilia was frequently but not constantly seen in these subjects. It was not well correlated with changes in eosinophils or lymphocytes in time or degree. It seems likely that the neutrophilia was a result of hormone administration, although it does not appear to be a good measure of adrenal cortical activity. In terms of numbers of cells, however, the changes in neutrophils are by far the most prominent hematologic alteration, so that leukocytosis was noted frequently. The mechanism producing neutrophilia following ster- oid administration is entirely obscure ; it should be pointed out that no increase in immature granulocytes occurred during the period of observation.

Although many of the subjects showed a decrease in monocytes at the same time that eosinopenia and lymphopenia were noted, this change in monocytes was difficult to evaluate since similar changes were noted in individuals observed throughout the day who did not receive steroids. No significant alterations in basophils were noted, nor were definite changes in volume of packed red cells or platelets seen in the few individuals in whom these measurements were followed.

Under the conditions of our experiments the intramuscular administration of compound $F$ acetate did not produce significant changes in steroids or cells whereas such changes were seen following the intramuscular administration of the free alcohol. This phenomenon may be due to the fact that the free compound was either dissolved or suspended in ethanol, whereas the acetate was in a saline-suspending medium. The fact that the suspending medium is of importance in absorption of steroids from intramuscular sites is demonstrated by the observation that free cortisone produced good effects when administered in ethanol, but not when administered in saline. It is also possible that the presence of the acetate group in the compound results in delayed absorption and utilization. Further studies are under way to elucidate this problem of absorption and hydrolysis of the ester. It should be emphasized that these studies of the usual steroid acetate suspensions for intramuscular injection were carried out by the use of single large doses and that the effect of repeated administration was not tested. It is quite possible that repeated administration might alter the steroid levels as well as cellular relationships. On the other hand, the relatively slow clinical effect of intramuscular cortisone acetate in contrast to its oral administration is no doubt related to the pharmacological implications of these studies.

Compound $\mathrm{S}$ given as the acetate or free alcohol was ineffective in producing changes in either steroid levels or cells, by either route of administration. Since this compound was prepared in a different physical form from the compounds $E$ and $\mathrm{F}$, it is possible that differences in particle size 
may play a role in its failure to increase steroid levels.

\section{SUMMARY AND CONCLUSIONS}

1. The administration by mouth of cortisone, cortisone acetate, compound $F$ (Kendall) and compound $F$ acetate to normal human subjects regularly resulted in an elevation of blood levels of 17-hydroxycorticosteroids, and in neutrophilia, lymphopenia, and eosinopenia. The administration of cortisone and compound $\mathrm{F}$ intramuscularly in alcoholic solution produced similar responses. The administration of compound $F$ acetate intramuscularly did not result in these changes, whereas the administration of cortisone acetate produced them irregularly. No significant changes followed the administration of compound $\mathrm{S}$ or compound $S$ acetate either orally or intramuscularly.

2. The maximum elevation of blood 17-hydroxycorticosteroid levels occurred approximately one hour after administration of the compound by either route. The maximum change in blood cells took place four to eight hours after administration.

3. Changes in eosinophils and in lymphocytes following steroid administration appeared to be closely related to each other, whereas changes in neutrophils, although generally greater in magnitude, appeared not to be closely related to other cellular changes either in time or degree.

4. The cellular changes that occurred following administration of these steroids appeared to be closely related to dosage and plasma 17-hydroxycorticosteroid levels.

\section{ACKNOWLEDGMENTS}

We are grateful to Drs. J. M. Carlisle and E. Alpert of Merck and Company, and to Dr. P. L. Julian of the Glidden Company for generous supplies of steroids. We wish to thank the Misses Doris Kurth, Betty Tatting and Carma Darley and Messrs. E. Myles Glenn and David S. Taylor for technical assistance.

\section{REFERENCES}

1. Dougherty, T. F., and White, A., Influence of hormones on lymphoid tissue structure and function. The role of the pituitary adrenotrophic hormone in the regulation of the lymphocytes and other cellular elements of the blood. Endocrinology, 1944, $35,1$.

2. Hills, A. G., Forsham, P. H., and Finch, C. A., Changes in circulating leukocytes induced by the administration of pituitary adrenocorticotrophic hormone (ACTH) in man. Blood, 1948, 3, 755.

3. Nelson, D. H., and Samuels, L. T., A method for the determination of 17-hydroxycorticosteroids in blood: 17-hydroxycorticosterone in the peripheral circulation. J. Clin. Endocrinol., 1952, 12, 519.

4. Porter, C. C., and Silber, R. H., A quantitative color reaction for cortisone and related 17,21-dihydroxy20-ketosteroids. J. Biol. Chem., 1950, 185, 201.

5. Randolph, T. G., Blood studies in allergy. I. The direct counting chamber determination of eosinophils in propylene glycol aqueous stains. J. Allergy, 1944, $15,89$. 